\title{
Frontières
}

\section{Tatouages, perçages et autres modifications corporelles}

\section{La gérance des gains, pertes et deuil associés au passage à la vie adulte}

\section{Jocelyne Thériault}

Volume 18, numéro 2, printemps 2006

La mort dans tous ses états

URI : https://id.erudit.org/iderudit/1073224ar

DOI : https://doi.org/10.7202/1073224ar

Aller au sommaire du numéro

Éditeur(s)

Université du Québec à Montréal

ISSN

1180-3479 (imprimé)

1916-0976 (numérique)

Découvrir la revue

Citer cet article

Thériault, J. (2006). Tatouages, perçages et autres modifications corporelles : la gérance des gains, pertes et deuil associés au passage à la vie adulte. Frontières, 18(2), 63-68. https://doi.org/10.7202/1073224ar

\section{Résumé de l'article}

Dans cet article, il est question des pratiques de modifications corporelles, comme le tatouage et le perçage, chez les adolescents. L'argumentation proposée est la suivante : dans nos sociétés modernes, l'absence de repères (psychologique et sexuel) propres à l'adolescence se double de l'absence de repères sociaux en général. Le marquage corporel apparaît ainsi comme un moyen privilégié de sacralisation du corps et d'affirmation de l'individualité. 


\section{Résumé}

Dans cet article, il est question des pratiques de modifications corporelles, comme le tatouage et le perçage, chez les adolescents. L'argumentation proposée est la suivante: dans nos sociétés modernes, l'absence de repères (psychologique et sexuel) propres à l'adolescence se double de l'absence de repères sociaux en général. Le marquage corporel apparaît ainsi comme un moyen privilégié de sacralisation du corps et d'affirmation de l'individualité.

Mots clés: tatouage - perçage modifications corporelles adolescence - deuil.

\section{Abstract}

This article is about the practices of physical modifications, as tattoo and piercing, amongst teenagers. According to the author's argumentation, already disoriented by their journey from child to adult, the teenagers find themselves in disoriented societies. Therefore, physical modifications would be a way for teenagers to sacralize their body and to assert their own individuality.

Keywords: tattoo - piercing - physical modifications - adolescence mourning.

\section{Tatouages, perçages et autres modifications corporelles La gérance des gains, pertes et deuil associés au passage à la vie adulte}

\section{Jocelyne Thériault, Ph. D., professeure, Département de sexologie, UQAM.}

Les pratiques de marquage corporel sont légion chez les adolescents et jeunes adultes des pays occidentaux. Elles ne caractérisent plus uniquement les jeunes de certaines classes sociales ni ceux d'appartenances institutionnelles spécifiques. Les tatouages et autres marquages corporels ne sont plus uniquement portés, par exemple, par les prisonniers, les marins ou les jeunes hommes des classes sociales défavorisées. Les pratiques de marquage corporel se sont démocratisées et universalisées. On estime, par exemple, que 10 à 15 pour cent des jeunes sont tatoués (Armstrong et al., 2002) et/ou percés et qu'un nombre toujours plus important d'entre eux s'adonnent à des automutilations corporelles (Brickman, 2004; Favazza, 1998, 1996).

Regroupant une multitude de pratiques et de techniques - de nature, intensité et étendue fort variées (Brickman, 2004) -, les modifications corporelles ont pour but premier de «travailler» le corps pour le modifier, l'inscrire, le maintenir ou le thématiser dans un sens particulier (Crossley, 2005). Elles témoignent donc d'une intention particulière. Examinées dans le contexte des enjeux psychosociaux et sexuels qui marquent le passage à la vie adulte, ces pratiques de modifications corporelles surviennent au moment où les jeunes de nos sociétés modernes sont confrontés à deux problèmes de taille: celui de la perte de repères psychologiques et sexuels qui marquent le développement normatif de l'adolescent et celui de la perte de repères sociaux qui marquent la vie d'aujourd'hui en société. C'est sur cette double affirmation que reposent les prémisses du présent article.

Afin d'examiner la portée et l'utilité de cette affirmation, deux perspectives théoriques seront utilisées ici. Les multifonctionnalités du corps marqué des jeunes de nos 
sociétés contemporaines seront interprétées suivant une lecture psychanalytique et une lecture sociale du marquage corporel. Loin de renforcer les oppositions qui séparent ces deux champs interprétatifs, une tentative d'intégration de ces différentes lectures sera faite.

À noter que les modifications corporelles ne peuvent être ramenées à une explication monolithique - les jeunes s'automutilant gravement ne pouvant être comparés à ceux décorant leurs corps de quelques tatouages légers. Notre étude portera sur les pratiques à "gravité moyenne». Sans être anodines, ni extrêmes (des pratiques dont les effets sont nuisibles et en profondeur), ces pratiques «de gravité moyenne» participent toutes d'un effort répété visant à modifier le corps.

\section{LES AUTOMUTILATIONS}

Parmi les pratiques d'automutilation, celles consistant à s'entailler la surface de la peau sont classées parmi les delicate self-mutilation. Poussés par un besoin compulsif de se couper, certains jeunes en ressentiraient une expérience de catharsis émotionnelle, du self purifying. En effet, cette pratique - en co-occurence avec les problèmes alimentaires dans 50\% des cas (Favazza et al., 1989) - modulerait l'état d'angoisse, de tension sexuelle, de colère et ou les états de vide dissociatifs ressentis et elle procurerait à ceux qui la pratiquent une sensation quasi physique de soulagement (Cross, 1993). Les études médicales traditionnelles (incluant les études psychanalytiques) sur les automutilations de type coupures ont, plus que toutes autres, mis l'accent sur l'aspect répressif, voire régressif de ces pratiques. On y voit une manifestation de refus, d'opposition, voire de pulsion de mort retournée contre soi. Cette forme de masochisme, différente du masochisme primaire, ferait en sorte que l'individu ne pourrait de façon satisfaisante diriger vers l'extérieur (vers les autres) la puissante force qui l'habite. Il resterait aux prises avec cette force impliquant ultimement la destruction, voire l'autodestruction.

Après avoir été pendant des années la seule perspective de poids à offrir pendant des années une explication au phénomène des automutilations corporelles, la psychiatrie traditionnelle (et occasionnellement la psychanalyse) voit aujourd'hui sa lecture du phénomène remise en question par certains. On lui reproche d'avoir assis ses résultats de recherche sur un biais de genre (biais d'échantillonnage) faisant en sorte que les automutilations aient été identifiées principalement chez les adolescentes et les jeunes femmes (Favazza, 1998). On lui reproche également d'avoir interprété les automutilations à la lumière des mythes persistants

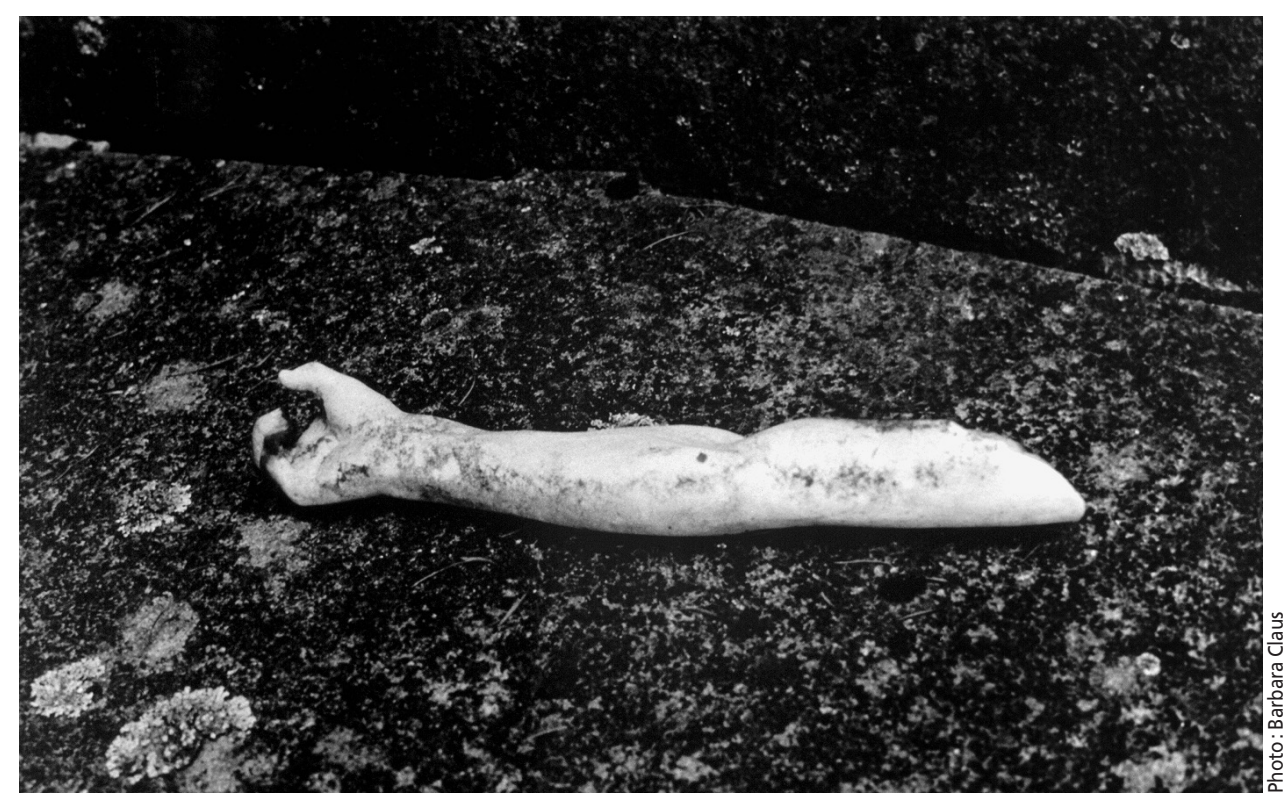

fondant les différences de genre, à savoir ceux-là mêmes à la base des stéréotypes rattachés au genre féminin: masochisme, passivité, infantilisme. Dans ce contexte, les automutilations à l'adolescence et à la jeunesse seraient interprétées comme un refus des responsabilités et des pressions sociales associées au passage à la vie adulte (Brickman, 2004). Une dénégation des conflits pubertaires se profilerait derrière cette attitude. On a également interprété cette pratique comme un refus (ou incapacité subjective) d'opérer les deuils de l'enfance, voire en particulier le deuil du lien infantile qui relie le jeune à ses parents.

D'autres auteurs plus contemporains interprètent la «prépondérance» chez les femmes des pratiques d'automutilation en se référant à l'hypothèse voulant que les expériences corporelles des femmes soient plus ambiguës que celles des hommes et que les femmes sont plus connectées à leur corps que les hommes (Cross, 1993). Dans ce contexte, on insiste moins sur les conflits pubertaires déniés par les jeunes que sur l'imposante somme de changements physiques (dans la forme du corps) vécus par les filles à la puberté, lesquels changements de contours ont des effets profonds sur leurs sensations et fantaisies en lien avec le corps. On affirme ici que les images corporelles seraient fragmentées: certains aspects du corps, surtout les seins, seraient perçus comme étrangers au moi (Cross, 1993, p. 44). Cet état de fait aurait des répercussions sur la façon dont les filles conçoivent leur relation à l'autre sexe. Les perceptions qu'elles ont des relations hétérosexuelles seraient ainsi interprétées à la lumière de leurs propres sensations et fantaisies sexuelles, voire mystérieuses, frustrantes et peu ou pas gérables (Kestenberg, 1975, dans Cross, 1993). Cette impression d'ab- sence de «contrôle»sur leur corps (et les relations), source d'inconfort, amènerait les jeunes femmes à chercher une solution à cet inconfort. La solution trouvée serait pour le meilleur ou le pire. Selon les auteurs ici concernés, la seule façon pour la fille de sentir qu'elle possède son corps - enjeu majeur du développement féminin - et vraisemblablement d'être ainsi à l'abri des pratiques d'automutilation et autres pratiques signant une difficulté de transition à la vie adulte, serait de parvenir à négocier les paradoxes qui l'habitent et les profonds changements physiques qui caractérisent son développement pubertaire, et ce, sans les nier ou sans être dépassés par eux. Toutefois, la gérance de ces paradoxes et l'intégration des nombreux changements pubertaires au moi n'irait pas de soi (Brooks-Gunn et Graber, 1999).

Doctors (1979, dans Cross, 1993) explique la prépondérance apparente des femmes à se tourner vers les automutilations par un argumentaire d'ordre social. Les filles et les femmes seraient confrontées à des messages contradictoires venant de leur culture. En effet, la fille recevrait à la puberté un ensemble de messages culturels contradictoires: être autonome mais non menaçante, s'accomplir tout en s'effaçant (self-denying), être sexuellement libérée mais "pure». Selon l'auteur, devant ces messages contradictoires, les femmes tenteraient de traiter les dilemmes perçus à l'intérieur des limites étroites de leurs corps plutôt qu'à l'extérieur d'elles, à défaut de défis sociaux où s'investir. Bien que passablement valable, cette théorie ne reçoit pas l'aval des chercheurs et des féministes contemporains. Cross (1993) avance l'idée que les pratiques d'automutilation (self cutters) et de désordres alimentaires, aussi bizarres, mortifères et/ou autodestructives 
qu'elles semblent être, sont néanmoins des tentatives faites pour prendre possession de son corps, pour le percevoir comme étant: 1) soi et non autre, 2) objet connu et maîtrisé (non uncharted et imprévisible) et 3) entité impénétrable (non invalide et contrôlé par l'extérieur). Ironiquement, ces luttes pour se "posséder» risquent chez certaines de perpétuer et d'exacerber des vulnérabilités vécues antérieurement, vulnérabilités en lien avec le soi physique. Selon la lecture qu'en fait la psychiatrie, ces vulnérabilités référeraient ultimement à un déficit du moi installé durant l'enfance chez certains individus automutilés. Ce déficit les aurait amenés, plus jeunes, à éprouver de la difficulté à identifier, exprimer verbalement et réguler toutes formes de tension physique (Favazza et al., 1989). Une telle difficulté aurait hypothéqué leurs relations précoces avec le soi, avec le corps, incluant le corps sexuel. Des états de tension extrême, de grand vide intérieur et/ou de quasi mort psychique auraient été vécus, sans que la personne ne puisse parvenir à les communiquer, à se calmer ou à s'habiter, faute d'un moi à l'abri d'un déficit, faute d'un moi relativement fonctionnel. À la puberté, période normative de grands bouleversements, tant physiques et sociaux qu'identitaires, les filles fragilisées par un déficit du moi verraient dans les pratiques d'automutilation (et dans les désordres alimentaires) un moyen d'exprimer les peines et vulnérabilités psychologiques vécues. Les douleurs psychiques ou le vide intérieur ressentis seraient convertis en blessures physiques par les automutilations, lesquelles, une fois transformées, seraient plus faciles à gérer, à regarder, à apaiser. Cette transformation ne serait pas porteuse d'un impératif de destruction mais bien plutôt d'un projet de délivrance.

Alors que d'aucuns auront vu dans les pratiques d'automutilation une plus grande tendance à retourner contre soi les pulsions de mort, voire à retourner contre soi sa colère, d'autres considèrent que les cicatrices des jeunes automutilés reflètent une certaine forme de narcissisme. On y réfère en parlant de la fonction décorative de la marque, preuve de courage chez les jeunes (Favazza et al., 1989).

Cross (1993) soutient que la première explication ne tient pas la route. Si les femmes étaient plus enclines à retourner contre elles leur agression, pourquoi les formes plus sévères d'automutilation se retrouvent-elles plus fréquemment chez les hommes (p. ex., les suicides)?

Brickman (2004) soutient que le discours médical aurait participé à la pathologie de la femme, tant celle au travail que sa fille automutilée. Selon certains, c'est leur corps - voire leur sexualité- non disci- pliné, non contrôlé, qui serait à l'origine des maux tels que le masochisme, la passivité et l'infantilisme. Ce corps serait à discipliner et à contrôler pour ne pas tomber dans les pièges de la pathologie en général et de l'automutilation en particulier. Une telle lecture des automutilations tend, selon l'auteur, à minimiser et à masquer les avancées faites par les femmes dans leur mouvement de libération et de promotion de leurs droits. D'énormes pressions sociales seraient déployées pour maintenir le statu quo, filtrer, voire gommer les avancées du féminisme dans la vie quotidienne des hommes et des femmes de nos sociétés. Ces efforts auraient été déployés tant du côté des filles que de leurs parents. N'a-t-on pas eu tendance, par exemple, à rendre les mères qui s'investissent sur le marché du travail responsables des problèmes d'automutilation de leurs filles?

Certes le discours médical a évolué et a tenté de dépasser les biais de genre rencontrés fréquemment dans les recherches sur les automutilations effectuées par le passé. Toutefois, Brickman (2004) avance que le mythe de la "female cutter» reste toujours fort présent dans les esprits et qu'il influence tant les productions populaires que les écrits scientifiques en médecine. On s'interroge sur les raisons qui font que le discours médical perpétue toujours un discours conservateur quant aux genres (Brickman, 2004). Dans un autre ordre d'idées, on s'interroge sur les raisons qui font que les jeunes de nos sociétés occidentales dites modernes en viennent à utiliser de telles pratiques «exutoires» pour exprimer ce qu'ils ont à exprimer, que ce soit nommable ou innommable. Seraient-ils entendus s'ils pouvaient nommer? Ontils été «trop» écoutés ou plutôt «trop» satisfaits dans leur exploration des plaisirs primaires? Ont-ils été suffisamment contenus dans leur exubérance infantile? Notre société moderne a-t-elle offert à leurs parents suffisamment de structures pour leur permettre de se sentir eux-mêmes suffisamment contenus dans l'exercice de leurs rôles de parents et de citoyens? Ce sont là autant de questions laissées sans réponse pour l'heure.

\section{LES TATOUAGES ET PERÇAGES}

Les perçages et tatouages corporels modernes sont devenus question d'esthétique au fil du temps tant leur présence est courante sur la peau des jeunes de nos sociétés. Thériault (1998, 2003, 2005) analyse les pratiques de tatouage et perçage répétées chez les jeunes dans la perspective du développement psychosexuel typique à l'adolescence et à la jeunesse. Tentant de faire le pont entre les perspectives psychiatriques, psychanalytiques et sociales, elle identifie deux paradoxes majeurs du développement psychosexuel adolescent qui semblent intervenir dans ces pratiques de modification corporelle et elle les analyse à la lumière d'un des phénomènes contemporains nouveaux accompagnant l'évolution des mœurs dans nos sociétés, à savoir l'absence d'interdits sexuels formulés par nos sociétés à l'endroit des jeunes (Marcelli, 1985).

Devant l'absence d'interdits sexuels à braver, phénomène sociétal inusité dans nos sociétés modernes aux origines judéochrétiennes, les jeunes auraient à trouver d'autres outils que ceux utilisés par leurs parents (outils servant souvent à structurer une personnalité à coloration névrotique) pour les aider à gérer les paradoxes normatifs de l'adolescence.

Les pratiques de marquage corporel seraient une solution des temps modernes aux paradoxes anciens de sexualisation et de différenciation associés aux tâches normatives du développement psychosexuel des jeunes (Thériault, 1998, p. 44). Le premier paradoxe oppose deux courants ou systèmes motivationnels - tendresse et sexualité; inhibition et exhibition de la sexualité-, lesquels courants devant éventuellement être intégrés au sein de la même personne pour qu'elle soit un jour capable d'amour véritable (Freud, 1987). Le second paradoxe oppose les tendances de connexion aux parents et/ou de séparation, l'affirmation de soi et/ou la reconnaissance de l'autre, lesquels courants devant être balancés, équilibrés pour éviter les pièges de la soumission à l'autre ou de la domination de l'autre (Benjamin, 1992; Thériault, 1998). Or depuis toujours, tant dans les écrits psychanalytiques que dans les écrits des sciences sociales, on tend à montrer que la gérance des conflits de l'adolescence ne va pas de soi. Par exemple, se construire une nouvelle identité sexuée et sexuelle à l'adolescence n'est pas une mince tâche. Ce développement interpelle tant les institutions sociétales que les relations interpersonnelles et les expériences intra-personnelles (Brooks-Gunn et Graber, 1999). Il implique que le jeune intègre en un tout au sein de son moi l'ensemble des changements vécus depuis la puberté, voire les changements physiques/sexuels, les changements cognitifs lui donnant accès à l'abstraction et les changements familiaux et sociaux dictés par la maturation cognitive et sexuelle. Cette intégration comporte à la fois des gains et des pertes pour que le jeune en vienne à se voir autonome et mature. Elle requiert l'abandon des liens d'attachement aux parents qui caractérisent l'enfance. Dans ce contexte, le jeune a à se séparer psychologiquement de ses parents, à se détacher de ses attachements infantiles 
au père et à la mère pour qu'un jour il ou elle puisse être capable d'intimité véritable et satisfaisante avec un ou une partenaire romantique (Blos, 1967; Benjamin, 1992; Brooks-Gunn et Graber, 1999; Gill et al., 2005). Cette intégration implique aussi l'abandon du soi infantile, réel et fantasmé. Elle implique quantité d'efforts pour se voir et être reconnu comme être sexué et sexuel et réussir à se faire reconnaître comme tel à l'intérieur de la famille et de la société. Cela ne va pas de soi. Faire le deuil des «parents tout-puissants», de la «famille refuge» pour aller vers d'autres formes d'organisation sociale n'est pas une mince tâche non plus. Sentir une appartenance à ce nouveau groupe serait un accomplissement complexe. Toutes ces finalités développementales impliqueraient non seulement des gains mais des renoncements importants, lesquels sont sources de douleurs, d'insécurités et de vulnérabilité pour l'adolescent et l'adolescente.

Il y a à peine 50 ans, la gérance de ces finalités était supportée par le social. Aujourd'hui il en va autrement, semble-t-il. L'individu est davantage laissé à lui-même (Giddens, 1991). Il se tournerait alors vers son corps pour le pousser aux extrêmes, le tester, etc.

Dans les sociétés dites plus «primitives», le corps des jeunes sert d'outil de transmission des valeurs sociales et de réalisation des tâches développementales menant à la maturité. C'est au moyen des rites de passage et des rites d'initiation que s'opérerait la structuration souhaitée. Selon certains auteurs, ces rites initiatiques permettent un rapport à la socialisation et un rapport à la vie et à la mort. Basquin (1983) par exemple rappelle que le rite d'initiation suppose un meurtre, la mise à mort de l'enfant que l'on a été, voire de l'enfant réel et rêvé, par soi et par les autres. Ces rites supposent le deuil de la mère primitive, la perte, voire la séparation d'avec cette mère de l'enfance. Ils supposent également le deuil, chez le jeune, de sa sexualité prégénitale, laquelle n'est ni franchement hétérosexuelle ni franchement homosexuelle mais est mue plutôt par les aléas d'une bisexualité psychique marquée par la toute-puissance. Chez les jeunes initiés de ces sociétés, le rite leur permet, en transformant leur corps (stigmates et marques sur le corps), d'accéder à un autre statut sexuel, voire à un corps sexué et sexuel autre. Un tel gain en entraînerait un autre: «L'individu confirmé dans sa masculinité ou féminité, tant pour soi que par la société, chercherait alors à entrer en lien intime avec l'autre corps sexué et sexuel» (Thériault, 1998, p. 40). L'engagement dans la vie adulte, institutionnalisé par la société, allait alors quasi de soi pour ces jeunes.

Qu'en est-il de ces rites dans nos sociétés dites modernes? Certains verront dans les pratiques contemporaines de marquage corporel de tatouage et perçage, dans les sports extrêmes et autres conduites ordaliques une version «moderne» des rites d'initiation déployés dans les sociétés plus traditionnelles. Bien que des parallèles aient été établis entre ces deux formes de pratiques (voir Thériault, 1998), il n'en demeure pas moins que l'accès via les épreuves du rite à un nouveau statut (statut d'adulte) - ce qui est le propre du rite initiatiquesemble incomplet chez les jeunes de nos sociétés qui ont recours aux pratiques de marquage corporel. Bien que les pratiques de marquage corporel semblent viser chez le jeune l'affirmation 1) de son individualité, de son identité propre via ce corps nouveau idiosyncratiquement décoré et marqué; 2) de sa capacité à se séparer des parents et des pratiques de ses parents, et 3) de son autonomie corporelle à faire ce qu'il veut avec son corps, elles ne semblent pas pour autant signer automatiquement son accession véritable à un statut nouveau. Des angoisses et des incertitudes seraient toujours opérantes chez plusieurs de ceux marqués de perçages et tatouages répétés (Thériault, 2003). Ces angoisses traduiraient vraisemblablement la présence d'un danger subjectif vécu au plan personnel chez ces jeunes, danger relié à l'intégration insuffisante des gains et pertes associés au passage de l'adolescence à la vie adulte (Thériault, 2003, 2005).

\section{FONCTIONS SOCIALES \\ ET MODIFICATIONS CORPORELLES}

Aujourd'hui le corps des jeunes serait à nouveau ciblé par le social, et ce, non plus pour le structurer, le soutenir comme ce fut le cas à l'époque des sociétés plus primitives mais pour d'autres fins (Gill et al., 2005). Le social dans nos sociétés modernes s'étant désacralisé pour reprendre la formulation de Giddens (1991), on assiste à une érosion des grandes certitudes politiques et, conséquemment, à un accroissement de l'individualisme et du consumérisme (Gill et al., 2005). C'est dans ce contexte socio-«politico »-psychologique que logerait le phénomène de marquage corporel contemporain. Laissé seul et sans guides pour construire un système de valeurs signifiantes capable de donner sens à sa vie, l'individu en général

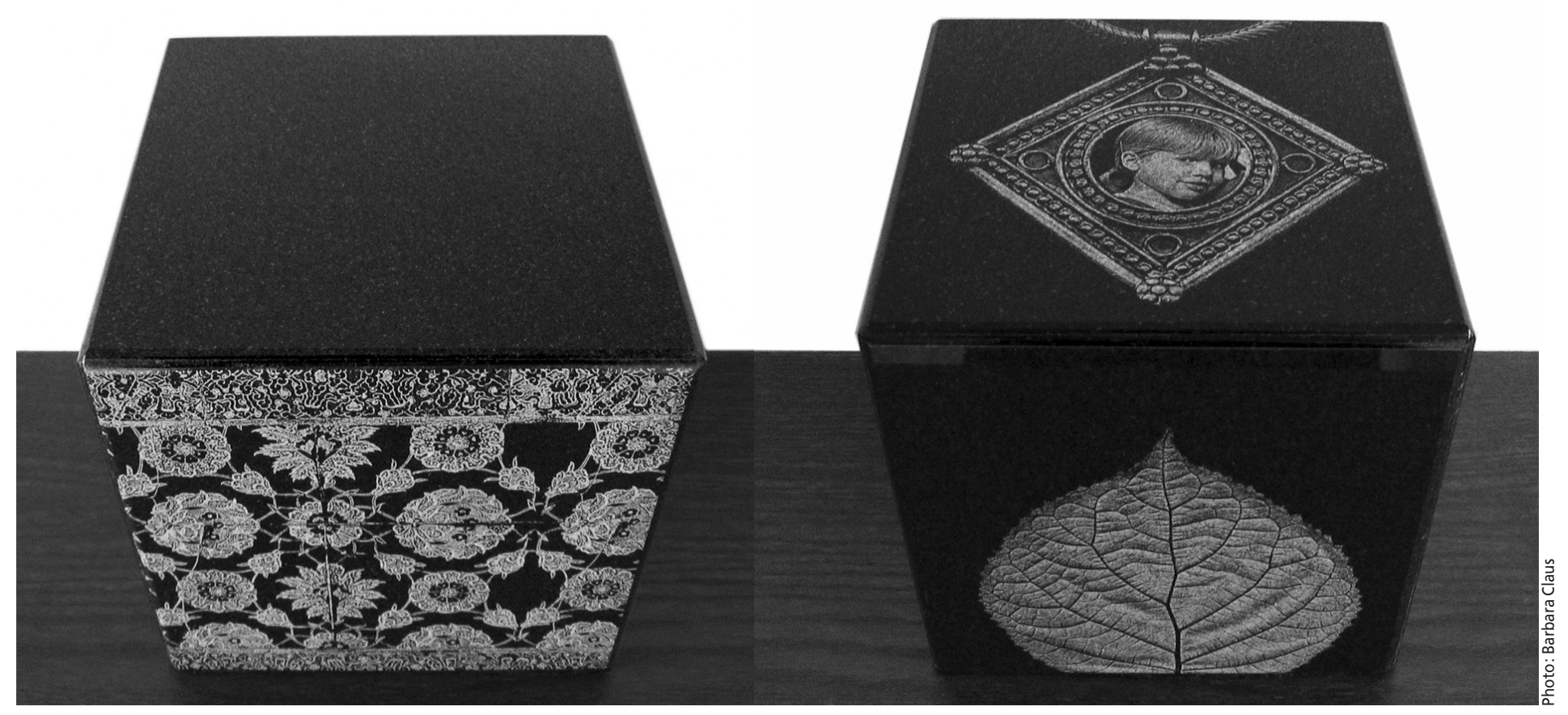


et le jeune marqué corporellement en particulier porterait maintenant une identité ancrée au corps. La peau marquée donnerait temporairement au jeune et à son environnement la preuve qu'il est compétent pour tolérer la douleur du marquage corporel. De là, il serait facile pour le ou la jeune de s'imaginer qu'il ou elle se sent capable de se distinguer des pratiques de ses parents et qu'il/elle est capable de s'individualiser (Thériault, 1998). Ces aspirations sont un leitmotiv retrouvé chez une majorité de gens marqués (Gill et al., 2005). Les tatouages inscrits dans la chair, les bijoux insérés dans la peau, participeraient donc chez le jeune d'un besoin de faire preuve de ses compétences (Thériault, 1998). Mais voilà qu'une telle préoccupation réflexive quant à son identité et à son corps propre - lot d'une dissolution graduelle de la tradition - serait, de l'avis de Giddens (1991), accompagnée d'une insécurité ontologique. Faire de son corps l'outil premier de la démonstration de sa compétence personnelle à se contrôler, à gérer pour le mieux ce qui arrive, à s'individualiser peut devenir essoufflant. Les pratiques de marquage corporel privilégiées par les jeunes de nos sociétés modernes ne permettraient pas d'atteindre automatiquement les mêmes visées que les rites initiatiques du passé car elles semblent privées du substrat social requis pour leur donner sens et en institutionnaliser les fonctions. Elles participeraient plutôt d'un body project dicté par le social.

Shilling (1993) dira que les significations du corps se sont privatisées. Dans une société de consommation, le corps devient source de capital symbolique. Le corps est jugé moins par ce qu'il fait que par ce dont il a l'air (Gill et al., 2005). On parle de fétichisation du corps, des muscles, de la peau. Turner (1984) interprète ce phénomène en termes d'éveil d'une société somatique. Les sociétés occidentales feraient pression pour que leurs membres, surtout les jeunes, «travaillent» leur corps, le transforme et en fasse un «projet» «non fini» (Gill et al., 2005). Un tel accomplissement quasi utopique, dicté par le social, serait défini comme faisant partie intégrante de l'identité individuelle. Dans un tel contexte, le corps en tant que source première d'identité verrait sa surface constituer un projet en développement. Faisant écho aux résultats de l'étude qualitative de Thériault (2003) réalisé auprès de jeunes femmes tatouées et percées à répétition, ceux de Gill et al. (2005) obtenus auprès de 140 jeunes hommes anglais testent la précédente affirmation et mettent en relief le fait que les modifications corporelles attestent d'une démarche identitaire et d'un effort de structuration personnelle. Les fonctions premières des modifications corporelles se

ALORS QUE D'AUCUNS AURONT VU DANS LES PRATIQUES

D'AUTOMUTILATION UNE PLUS GRANDE TENDANCE

À RETOURNER CONTRE SOI LES PULSIONS DE MORT,

VOIRE À RETOURNER CONTRE SOI SA COLÈRE, D'AUTRES CONSIDÈRENT QUE LES CICATRICES DES JEUNES AUTOMUTILÉS

REFLÈTENT UNE CERTAINE FORME DE NARCISSISME.

liraient comme suit: se sentir différent (individualisme, affirmer son autonomie, rébellion); exprimer une attitude personnelle libertaire et une autonomie du corps (faire tout ce que l'on veut de son corps, preuve ultime d'indépendance et d'autonomie); refléter un corps moralement responsable (le corps est une entité qui doit être disciplinée et on se sent la responsabilité de le faire). Ce dernier phénomène fut interprété en termes d'attitude hautement moralisante chez les jeunes hommes s'adonnant aux modifications corporelles (Gill et al., 2005) et en termes de contrôle personnel exercé sur le corps chez les jeunes femmes s'adonnant au marquage corporel répété (Thériault, 2003). On y distingue soit un besoin de censurer les transgressions pour écarter la culpabilité (Gill et al., 2005), soit un besoin de contrôle répété sur le corps par l'inscription «régulière » de nouveaux perçages et tatouages sur la peau pour écarter l'angoisse (Thériault, 2003). Dans ce dernier cas, tout se passe comme si les raisons pour lesquelles les pratiques de marquage corporel avaient été instaurées à l'origine avaient échouées, à tout le moins partiellement, et qu'il fallait les répéter pour tenter encore et encore d'endiguer cette angoisse.

Outil pour fournir des preuves de compétence, de contrôle exercé sur soi (compétence à contenir la douleur, à se contenir), le corps tatoué (peau symboliquement épaissie, renforcée par les tatouages et perçages) reprendrait sur lui ce que la société ne lui offre plus, soit des balises externes en matières de sexualité et de rattachement à une collectivité signifiante (Thériault, 1998). Tout en ornant et en «armant» la peau (frontière entre l'extérieur et l'intérieur), les marquages corporels seraient vraisemblablement utilisés pour endiguer les angoisses développementales qui, par le passé, étaient endiguées par le social qui, par exemple, imposait son lot d'interdits sexuels. Sans ces interdits, le ou la jeune doit se prouver qu'il ou elle est capable de dépasser ce qui l'assaille (et qui assaille les adolescents de tout temps: les désirs œdipiens resurgis, le deuil des figures infantiles impliquées dans ce conflit, le deuil de son moi infantile et des sources de plaisir d'antan). Mais avoir tant de preuves à fournir serait un processus essoufflant pour le narcissisme d'un jeune. À mesure qu'il ou elle tente d'endiguer certaines angoisses, d'autres prendraient le relais, faute d'un système social suffisamment structuré et sacralisé pour supporter l'adolescent ou l'adolescente dans sa démarche.

\section{CONCLUSION}

Conséquences vraisemblables de la désacralisation de la vie sociale à notre ère de «modernité», les pratiques de modification corporelle telles que le tatouage et le perçage contribueraient chez les jeunes à sacraliser le corps, à promouvoir l'individualisme (et non pas nécessairement l'individualisation) et le consumérisme (des pratiques à consommer) caractérisant les membres de nos sociétés modernes. Faute d'une société "resacralisée», faute d'une solution de rechange satisfaisante pouvant offrir aux jeunes un sens de sécurité, condition essentielle d'une démarche vers la maturité, il se peut que les pratiques de modification corporelle, fruit de notre société, non seulement se révèlent pour les jeunes une tentative de solution au problème social et développemental posé, mais qu'elles soient également sources de préoccupations et d'insécurités. Si le corps représente le principal, voire le seul lieu d'ancrage de l'identité, l'individu risque de se sentir menacé. Alors qu'auparavant les rôles que jouait l'individu (la position qu'il occupait dans la société) contribuaient grandement à la construction d'une identité plus stable et sécurisante, le corps propre s'appropriant maintenant les fonctions de contenant de l'identité risque de s'essouffler dans sa démarche pour fournir des preuves de compétence. Certes le corps marqué ou modifié signale une capacité de discipline, d'individualisme et d'autonomie. Il signale également un risque possible d'instabilité et d'insécurité face au corps vieillissant et à l'incapacité du corps à répondre à lui seul aux nombreuses demandes psychosexuelles, interpersonnelles et sociétales nourrissant le sentiment d'identité. 
À défaut de se confronter de façon satisfaisante aux nombreux deuils accompagnant la transition à l'adolescence et à la vie adulte, à défaut, ce faisant, de pouvoir opérer l'intégration nécessaire, au sein du moi, des nombreux changements normatifs qu'entraîne la maturation pubertaire (changements ou gains physiques/sexuels, cognitifs, sociaux/familiaux), l'individu pourra plus difficilement atteindre un degré suffisant de confort au plan identitaire. Il est possible de penser que cette difficulté persistera malgré les nombreuses compétences qu'il ou elle aura développées dans la maîtrise de son corps. À défaut d'un cadre social balisant ces changements psychosociaux et sexuels, à défaut de guidage et de support pour opérer les deuils nécessaires et intégrer les gains connexes, l'individu se retrouvera trop souvent seul face aux énormes défis que posent le passage à l'adolescence et le passage à la vie adulte.

\section{Bibliographie}

ARMSTRONG, M. L. et C. MCCONNELL (1994). "Tattooing in Adolescents: More Common Than You Think-The Phenomenon and Risks", Journal of School Nursing, vol. $10, \mathrm{n}^{\circ} 1$, p. 26-33.

ARMSTRONG, M.L., D.C. OWEN, A.E. ROBERTS et J.R. KOCH, (2002). «College student and tattoos. Influence on image, identity, family and friends", Journal of Psychosocial Nursing, vol. 40, $\mathrm{n}^{\circ} 10$, p. 20-29.
BASQUIN, M. (1983). «Les rituels à l'adolescence», Neuropsychiatrie de l'enfance, $\mathrm{n}^{\circ} 31$, p. 355-359.

BENJAMIN, J. (1992). Les liens de l'amour, Paris, Métailié.

BLOS, P. (1967). Les adolescents: essai de psychanalyse, Paris, Stock.

BRICKMAN, B. J. (2004). «"Delicate" cutters: Gendered Self-mutilation and Attractive Flesh in Medical Discourse», Body and Society, vol. $10, \mathrm{n}^{\circ} 4$, p. $87-111$.

BROOKS-GUNN, J. et J. A. GRABER (1999). "What's sex got to do with it? The development of sexual identities in adolecence », R.J. Castrada et R.D. Ashmore (dir.), Self social identity and physical health (p. 155181 ), Collection Rutgers, Series on self and social identity, vol. 2, New York, Oxford University Press, 209 p.

CROSS, L.W.(1993). «Body and self in feminine development: Implications for eating disorders and delicate self-mutilation ", Bulletin of Menningu Clinic, vol. 57, $\mathrm{n}^{\circ}$ 1, p. 41-68. Menningu Foundation.

CROSSLEY, N. (2005). "Mapping reflexive body techniques: On body modification and maintenance », Body and Society, vol. 11, $\mathrm{n}^{\circ} 1$, p. 1-35.

FAVAZZA, A.R., L. DE ROSEAR et K. CONTERIO, (1989). «Self-Mutilation and eating disorders », Suicide and Life-Threatening Behavior, vol. 19, n 4, p. 352-361.

FAVAZZA, A.R. (1996). Bodies under siege: Self-mutilation and Body modification in culture and psychiatry, Baltimore, MD, Johns Hopkins University Press.
FAVAZZA, A.R. (1998). «The coming of age of self-mutilation", The Journal of Nervous and Mental disease, vol. 186, $\mathrm{n}^{\circ}$ 5, p. 259268.

FREUD, S. (1987). Trois essais sur une théorie de la sexualité, Paris, Gallimard.

GIDDENS, A. (1991). Modernity and SelfIdentity: Self and Society in the Late Modern Age, Cambridge, Polity.

GILL, R., K. HENWOOD et C. McLEAN, (2005). «Body Projects and the Regulation of Normative Masculinity ", Body and Society, vol. $11, \mathrm{n}^{0} 1$, p. $37-62$

LE BRETON, D. (2002). Signes d'identité: tatouages, piercings et autres marques corporelles, Paris, Métailié.

MARCELLI, D. (1985). «Entre l'interdit et l'obligé: L'inhibition sexuelle à l'adolescence", Semaine des Hôpitaux de Paris, vol. $61, \mathrm{n}^{\circ} 27$, p. 1973-1976.

SHILLING, C. (1993). The Body and Social Theory, London, Sage.

THÉRIAULT, J. (1998). «Nouvel esthétisme, sexualité et intimité: réflexion préliminaire sur le corps tatoué et percé aux périodes de l'adolescence et de la jeunesse», Filigrane, vol. $7, \mathrm{n}^{\circ} 1$, p. 37-47.

THÉRIAULT, J. (2003). «Le marquage corporel et le désir de minceur chez la jeune femme: réflexion sur le contrôle et sur le corps à l'adolescence», dans J.J. Levy et al., (dir.). Santé et société: aspects psychosociaux, Québec, Presses de l'Université du Québec, p. 107-119.

THÉRIAULT, J. (2005) «Douleur physique du marquage corporel ", Adolescence, $\mathrm{n}^{\circ} 23$, p. 743-754.

TURNER, B.S. (1984). The Body and Society, Oxford, Blackwell. 